\title{
ENFOQUE DE LA GENERACIÓN DE CAPITAL INTELECTUAL DESDE LA GESTIÓN DE LA INVESTIGACIÓN EN LAS UNIVERSIDADES PÚBLICAS DE LA COSTA CARIBE DE COLOMBIA
}

\author{
Carlos Ramón Vidal Tovar ${ }^{1}$
}

Recibido: 17 de mayo de 2013

Aceptado: 12 de agosto de 2013

\section{Resumen}

El Objetivo general de esta investigación fue determinar el tipo de enfoque aplicado en la gestión de la investigación para la generación de capital intelectual en las universidades públicas de la Costa Caribe de Colombiana. La variable objeto de estudio fue sustentada en las teorías de Eduardo Bueno Campos (2003) y Antonio Cobo Jiménez (2006). Para tal efecto, se realizó una investigación aplicada, de tipo analítico situacional, no experimental, transversal y de campo, con una población de siete (7) universidades conformada por 290 personas y una muestra de 79 sujetos informantes, líderes de grupos de investigación categorizados y avalados por cada universidad objeto de estudio, a los cuales se les aplicó un cuestionario tipo Likert de 24 ítems, validado por siete expertos de la Universidad Rafael Belloso Chacín. La confiabilidad fue calculada a partir de una prueba piloto a veinte sujetos y se calculó el coeficiente de Alfa Cronbach, obteniendo un resultado de 0,952, lo cual es de una confiabilidad elevada. Los datos obtenidos fueron tratados estadísticamente a partir del cálculo de la media, la frecuencia absoluta y la frecuencia relativa. Los resultados evidenciaron que las universidades de la Costa Caribe Colombiana no tienen un enfoque definido para el desarrollo de la investigación y lo fundamentan tomando en cuenta el predominio de los enfoques estratégico corporativo y en menor medida el social evolutivo.

Palabras clave: Capital intelectual, universidad, investigación,

${ }^{1}$ Ingeniero de Alimentos, Especialista en Ingeniería de Procesos Industriales, Magíster Scientiarum en Ciencia 


\section{GENERATION APPROACH FROM INTELLECTUAL CAPITAL MANAGEMENT RESEARCH IN PUBLIC UNIVERSITIES OF THE CARIBBEAN COAST OF COLOMBIA.}

\section{Abstract}

The objective of this research was to determine the type of approach used in the management of research to generate intellectual capital in public universities in the Colombian Caribbean Coast. The variable under study was supported by the theories of Eduardo Bueno Campos (2003) and Antonio Cobo Jiménez (2006). To this end, we conducted applied research, analytical type situational, not experimental, and cross country, with a population of seven (7) universities comprised 290 people and a sample of 79 subjects informants, leaders of research groups categorized and endorsed by each university under study, to which was applied a questionnaire of 24 Likert items, validated by seven experts from the University Rafael Belloso Chacin. Reliability was calculated from a pilot trial to twenty subjects and calculated the Cronbach alpha coefficient, obtaining a score of 0.952 , which is a high reliability. The obtained data were statistically analyzed by calculating the average absolute frequency relative frequency. The results showed that universities in the Colombian Caribbean coast have no defined approach to the development of the underlying research and taking into account the prevalence of corporate strategic approaches and to a lesser extent social evolution.

Keywords: Intellectual Capital, University, Research.

\section{Introducción}

Desde 1992, la evolución de los modelos de capital intelectual ha sido constante y se pueden agrupar en función del tipo de enfoque predominante en cada uno de ellos: el Enfoque Financiero Administrativo (1992-1998), el Enfoque Estratégico-Corporativo (1997-2001), el Enfoque Social-Evolutivo (2000-2011) y otros en construcción. Cada uno de sus componentes permiten el análisis y medición del capital intelectual a partir de su contenido básico en torno a tres grandes bloques: capital humano (genérico y específico), capital estructural (organizativo y tecnológico) y capital relacional (de negocio o comercial y social o comunicacional).

Para las universidades colombianas es significativo e importante la visualización y reconocimiento de sus grupos de investigación en el Sistema Nacional de Ciencia, Tecnología e Innovación -
SNCTI, tomando como base los productos de investigación reportados, la calidad de estos y la madurez o trascendencia del grupo. Lo cual da a las universidades un status en el escalafón a nivel nacional y así acceder a mejores fuentes de financiación para la investigación por medio de sus grupos en diferentes convocatorias. El reconocimiento y transcendencia de grupos de investigación se genera por la visibilización de sus diferentes productos científicos.

Bajo el escenario planteado, las universidades de la Costa Caribe Colombiana como organización necesitan definir el enfoque necesario para la orientación de la investigación con el fin de desarrollar un conjunto de productos o activos intangibles y competencias en sus investigadores, es decir, Capital Intelectual; su organización con el entorno socio económico-productivo; los 
medios, herramientas y estructura física necesaria para aumentar la valoración de sus indicadores genéricos y específicos. El objetivo de esta investigación fue el determinar el tipo de enfoque aplicado en la gestión de la investigación para la generación de capital intelectual en las universidades públicas de la costa caribe de colombiana, para lo cual se fundamentó en los siguientes contenidos teóricos.

\section{Contenido}

\section{Capital Intelectual}

El Capital Intelectual acorde con Bueno (2003), corresponde al conjunto de activos intangibles que generan y generarán valor para la organización en el futuro como conocimientos poseídos por las personas, sus capacidades, talento, ideas, destrezas, invenciones, patentes, sistemas, aplicaciones y todo tipo de trabajo creativo, el reconocimiento de la sociedad, la calidad de las relaciones que se mantienen con miembros y equipos internos o externos a la organización, son algunos de los activos intangibles que explican buena parte de la valoración que la sociedad y comunidad científica conceden a una universidad o centro de investigación. es decir, todos aquellos activos intelectuales o de conocimiento de naturaleza intangible que se puedan identificar, definir, y medir; que son de uso específico, concreto de la organización e idiosincrásicos para el sujeto de conocimiento estudiado (Bueno, 2003).

En ese orden, la perspectiva global y estratégica de los activos intelectuales de una empresa se refiere a la suma del conocimiento de sus miembros y de la interpretación práctica del mismo. Es un fenómeno complejo, resulta de una práctica colectiva, que crea valor a través de sus relaciones e interrelaciones entre recursos, competencias y capacidades organizacionales (Da Silva Santos et al., 2009).
Los conceptos anteriores, permiten su integración con el fin de definir al capital intelectual como al conjunto de activos intangibles generados por la dinámica del conocimiento de las personas que conllevan a la generación de valor agregado o ventajas sostenibles para la Universidad; sus grupos o equipos de trabajo y al individuo como tal; las cuales no se reflejan en los estados financieros o contables pero si permiten su visibilidad y competitividad a largo plazo en la comunidad científica o el entorno donde se desarrollan.

\section{Componentes del Capital Intelectual}

Seguí (2007), afirma que la última década, ha generado numerosos modelos para la gestión del capital intelectual. El denominador común de ellos es la clasificación que realizan de los componentes del capital intelectual según su naturaleza. El capital intelectual representa la combinación de los recursos humanos, organizativos y relacionales de una empresa. De este modo, se puede descomponer el capital intelectual en: Capital humano, Capital estructural y Capital relacional. El capital intelectual es más que la suma de los componentes individuales, ya que la conectividad de los 3 elementos citados también puede ser fuente de valor para la organización.

En ese orden, Bueno (2003), agrupa los activos intangibles teniendo en cuenta su naturaleza en tres componentes: Capital Humano, Capital Estructural y Capital Relacional. Subdivide El Capital Estructural, en Capital Organizativo y Capital Tecnológico. Al Capital Relacional lo divide en Capital Negocio y Capital Social. Las razones para descomponer el Capital Estructural y el Capital Relacional se fundamentan en la propia evolución de los conceptos involucrados y por la observación de la emergencia de determinadas prácticas, consideradas como relevantes y pioneras en materia de Gestión del Conocimiento (Cobo, 2006). 
Aunado a lo anterior, se debe tener en cuenta la influencia indiscutible de la era digital o era de la economía digital. La cual, debido a la utilización de la Internet como medio de integración del Capital humano con el Capital relacional y el Capital Estructural, con el fin de crear valor por medio de la promoción de una nueva plataforma de competencia, extrayendo el poder de las redes de negocios o business webs ha ocasionado la inclusión de un componente más al capital intelectual denominado Capital Digital (Tapscott y otros, 2001). A continuación se definen como componentes principales del capital intelectual al capital humano, el capital estructural, el capital relacional y capital digital.

\section{Enfoques predominantes en el Capital Intelectual}

Cobo (2006) analiza lo propuesto por Bueno (2003), al reunir la tipología de los principales modelos que han intentado medir y gestionar al capital intelectual desde la aparición del primer informe de Skandia (1992) sobre el tema, hasta la fecha. Divide los modelos en tres enfoques predominantes en su evolución así:

\section{Financiero Administrativo.}

Se identifican con este enfoque todos los modelos propuestos entre 1992 a 1998, entre ellos se encuentran Navigator Of Skandia (1992), Technology Broker (1996), Canadian Imperial Bank of Comerce (1996), University Of Western Ontario (1996), Intangible Assets Monitor (1997), Edvinsson, L. y Malone, M.S. (1997), Stewart, T.A. (1997) y Dow Chemical (1998).

Es de clara influencia contable, los modelos identificados con esta propuesta responden a distintas demandas para medir e informar sobre el capital intangible o sobre los activos intangibles de las organizaciones que el mercado reconoce, pero no la contabilidad tradicional; presentan heterogeneidad y ausencia de lógica teórica (Bueno, 2008). En general, se utilizan los mismos modelos y metodologías para la gene- ración de informes de activos intangibles y para la gestión de dichos activos (Viedma, 2002).

\section{Estratégico corporativo}

En este enfoque se encuentran incluidos los modelos generados entre 1997 al 2001, forman parte de este enfoque los modelos Atkinson (1997), Roos (1997), Intelect (1997), Intellectual Capital Model (1998),Dirección Estratégica por Competencias: Capital Intangible (1998), ABCcluster del conocimiento (2000) e IBCS (2001).

Para Bueno (2008), los modelos generados con este enfoque inician el proceso de madurez del concepto capital intelectual; están centrados en una perspectiva estratégico-corporativa en los que se va produciendo una armonización de los componentes o «capitales» que lo integran, superando la heterogeneidad y ausencia de lógica teórica de la etapa inicial. Los modelos de este enfoque proponen una estructura organizativa formal e informal, a los métodos y procedimientos de trabajo, al software, a las bases de datos, a los sistemas de I+D (investigación y desarroIlo), a los sistemas de dirección y gestión, y a la cultura de la empresa.

\section{Social Evolutivo}

En este enfoque se incluyen los modelos desarrollados desde el año 2000 al 2005, en él se incluyen los modelos American Society For Training and Development «ASTD» (2000), NOVA (2000), KMCl (2001) el modelo Intellectus (2003).

En este enfoque los modelos presentan madurez del concepto y evolución en los componentes que lo constituyen son dinámicos como el Social, Cultural, de Innovación o de Emprendimiento, entre otros (Bueno, 2008). Más que identificar indicadores y modelos de medición, los modelos implementados bajo este enfoque identifican los activos que son estratégicamente importantes y que se encuentran contenidos en sus competencias clave, los que permiten a la compañía otorgar beneficios a los consumido- 
res, son únicas y agregan potencial para abarcar un mayor número de mercados.

En apoyo a lo anterior, Cobo (2006), clasifica los modelos de capital intelectual teniendo en cuenta la orientación de sus componentes así: en los modelos propuestos entre 1.992-1.998 los componentes o «Capitales» son no armonizados, toman como base los activos intangibles y competencias de la organización. En los modelos propuestos entre 1997 al 2001, sus componentes son armonizados, están constituidos por el capital Humano, capital Estructural y capital Relacional. A partir del año 2000 en adelante, los componentes son armonizados y han evolucionado debido a la dinámica de las organizaciones en su entorno.

\section{Metodología}

Se realizó una investigación aplicada, de tipo analítico situacional, no experimental, transversal y de campo, con una población de siete (7) universidades conformada por 290 líderes de grupos de investigación, tomando 79 sujetos informantes de grupos de investigación categorizados en el sistema nacional de ciencia, tecnología e innovación y avalados por cada universidad objeto de estudio,

Es importante acotar, que para lo anterior se aplicó un instrumento tipo cuestionario con una escala Lickert de cinco alternativas, Totalmente de Acuerdo (5), Parcialmente de Acuerdo (4),
Neutral (3), Parcialmente en desacuerdo (2) y Totalmente en desacuerdo (1); el instrumento fue validado por siete expertos de la Universidad Rafael Belloso Chacín. La confiabilidad fue calculada a partir de una prueba piloto a veinte sujetos y se calculó el coeficiente de Alfa Cronbach, con el paquete estadístico SPSS V.20.obteniendo un resultado de 0,952, lo cual es de una confiabilidad elevada.

Los datos obtenidos fueron tratados estadísticamente a partir del cálculo de la media, la frecuencia absoluta y la frecuencia relativa, utilizando la hoja de cálculo Excel de Microsoft Windows 2010, con el cual se generaron las tablas de frecuencia para cada indicador y los gráficos de frecuencia promedio para las dimensiones, los resultados fueron interpretados utilizando el siguiente Baremo: todas las respuestas elegidas como Totalmente de Acuerdo y Parcialmente de Acuerdo fueron consideras como presencia favorable con una frecuencia relativa entre 50 y $100 \%$; las respuestas Parcialmente en desacuerdo y Totalmente en desacuerdo fueron consideras como presencia desfavorable con una frecuencia relativa entre 0 y $49 \%$.

Las respuestas neutrales se analizaron tomando como base el valor de $15 \%$ correspondiente a la media obtenida de todas las respuestas neutrales dadas por los informantes. Indicadores con valores superiores a este fueron considerados estadísticamente significativos y analizados de forma particular para cada dimensión.

\section{Resultados}

Tabla 1. Frecuencia Absoluta y Relativa del Indicador Financiero Administrativo

\begin{tabular}{lcc}
\multicolumn{1}{c}{ ALTERNATIVA } & F. A. & F. R. \% \\
\hline 1 - Totalmente en Desacuerdo (TDA) & 13 & 17 \\
\hline - Parcialmente en Desacuerdo (DA) & 11 & 14 \\
3 - Neutral (N) & 16 & 21 \\
4 - Parcialmente de Acuerdo (PA) & 22 & 27 \\
\hline - Totalmente de Acuerdo (TA) & 16 & 21 \\
\hline
\end{tabular}


La tabla 1 permite apreciar que solo el $21 \%$ manifestó estar totalmente de acuerdo y el $27 \%$ parcialmente de acuerdo con el enfoque financiero administrativo acorde a la valoración de los productos intangibles obtenidos por la investigación en la universidad, el tener en cuenta los activos intangibles producidos por la investigación en los indicadores de crecimiento de la universidad y los informes periódicos presen- tados sobre los activos intangibles producidos por la investigación, lo cual corresponde a una presencia favorable del $48 \%$, mientras que el $17 \%$ contemplan estar totalmente en desacuerdo, el 14\% parcialmente en desacuerdo, dando como resultado una presencia desfavorable del $31 \%$. Por otro lado, es significativo para el $21 \%$ de informantes su actitud neutral frente a cada indicador valorado.

Tabla 2. Frecuencia Absoluta y Relativa del Indicador Estratégico Corporativo

\begin{tabular}{lcc}
\multicolumn{1}{c}{ ALTERNATIVA } & F. A. & F. R. \% \\
\hline 1 - Totalmente en Desacuerdo (TDA) & 11 & 14 \\
2 - Parcialmente en Desacuerdo (DA) & 10 & 12 \\
3 - Neutral (N) & 14 & 18 \\
4 - Parcialmente de Acuerdo (PA) & 23 & 29 \\
5 - Totalmente de Acuerdo (TA) & 21 & 27 \\
\hline
\end{tabular}

Fuente: Elaboración propia (2012).

Referente al indicador estratégico corporativo, la tabla 2 permite apreciar que un $27 \%$ de los líderes de investigación manifestaron estar totalmente de acuerdo y un $29 \%$ parcialmente de acuerdo al opinar sobre la integración en la misión de la universidad la importancia de la investigación como función sustantiva, la protección legal de los activos intangibles producidos por investigadores y el establecimiento de planes de mejoramiento orientados a la producción de capital intelectual desde la investigación, originando como resultado una presencia favorable del $56 \%$. Por otro lado, el $14 \%$ manifestó estar totalmente en desacuerdo, el $12 \%$ parcialmente en desacuerdo, para una presencia desfavorable del $26 \%$. Es significativa la valoración dada del $18 \%$ de actitud neutral.

Tabla 3. Frecuencia Absoluta y Relativa del Indicador Social Evolutivo

\begin{tabular}{lcc}
\multicolumn{1}{c}{ ALTERNATIVA } & F. A. & F. R. \% \\
1 - Totalmente en Desacuerdo (TDA) & 10 & 13 \\
2 - Parcialmente en Desacuerdo (DA) & 12 & 15 \\
3 - Neutral (N) & 16 & 20 \\
4 - Parcialmente de Acuerdo (PA) & 25 & 31 \\
5 - Totalmente de Acuerdo (TA) & 16 & 21 \\
\hline
\end{tabular}

Fuente: Elaboración propia (2012).

Se puede observar en la tabla 3 como el $21 \%$ de los líderes de grupos de investigación manifestaron estar totalmente de acuerdo y el $31 \%$ parcialmente de acuerdo al opinar sobre el indicador social evolutivo basado en la utilización por las universidades del capital intelectual producido por la investigación para darse a conocer en su entorno social, para captar recursos 
económicos y estudiantes, lo cual tiene una presencia favorable del $52 \%$.

En ese orden, un $13 \%$ manifestó estar totalmente en desacuerdo, un $15 \%$ parcialmente en desacuerdo evidenciando una presencia desfavorable del $28 \%$. Es significativa la actitud neutral del 20 .

\section{Discusión de resultados}

Los resultados anteriores evidencian diferencias en las opiniones de los líderes de investigación, lo que permite inferir que en algunas universidades de la costa caribe colombiana no aplican de forma regular un enfoque financiero administrativo en la investigación; lo que puede ser debido al desconocimiento de la valoración de los productos intangibles obtenidos por la investigación o el no tenerlos en cuenta en los indicadores de crecimiento de la universidad. Acorde con Bueno (2008), es un enfoque de clara influencia contable, donde se mide e informa sobre el capital intangible o sobre los activos intangibles de las organizaciones que el mercado reconoce, pero no la contabilidad tradicional.

En ese orden, se puede afirmar que el enfoque estratégico corporativo está presente en algunas universidades públicas de la Costa Caribe colombiana, pero en otras no se utiliza de forma estratégica los productos de investigación en su misión, la protección legal de los activos intangibles y el establecimiento de planes de mejoramiento para producir capital intelectual. Para Bueno (2008), es un enfoque de interrelaciones existentes entre los componentes de capital intelectual, los armoniza, los integra superando la heterogeneidad y ausencia de lógica teórica de la etapa inicial.

Referente al indicador social evolutivo, los resultados contradicen lo planteado por Bueno (2008), al afirmar que el enfoque social evolutivo corresponde a la resultante de la interac- ción evolucionada de los modelos, preocupado por componentes o capitales dinámicos como el social, cultural o de emprendimiento. Este fenómeno se enmarca en el concepto producido por Bueno (2008) sobre capital intelectual: «Acumulación de conocimiento que crea valor o riqueza cognitiva poseída por una organización, compuesta por un conjunto de activos de naturaleza intangible o recursos y capacidades basados en conocimiento, que cuando se ponen en acción, según determinada estrategia, en combinación con el capital físico o tangible, es capaz de producir bienes y servicios, de generar ventajas competitivas o competencias esenciales para la organización en el mercado».

\section{Conclusiones}

En algunas universidades de la Costa Caribe colombiana no aplican de forma regular un enfoque financiero administrativo en la investigación; lo que puede ser debido al desconocimiento de la valoración de los productos intangibles obtenidos por la investigación o el no tenerlos en cuenta en los indicadores de crecimiento de la universidad.

El enfoque estratégico corporativo está presente en algunas universidades públicas de la Costa Caribe colombiana, pero en otras no se utiliza de forma estratégica los productos de investigación en su misión, la protección legal de los activos intangibles y el establecimiento de planes de mejoramiento para producir capital intelectual.

En ese orden, se puede afirmar que en algunas universidades de la Costa Caribe colombiana existe la presencia del enfoque social evolutivo para la investigación. Pero en otras pueden desconocer cómo utilizar el capital intelectual producido por la investigación para darse a conocer en su entorno social, para captar recursos económicos y vincular nuevos estudiantes a sus programas de formación. 
Lo anterior permite apreciar como el enfoque financiero administrativo presenta una presencia desfavorable, mientras el enfoque estratégico corporativo y el social evolutivo poseen presencia favorable de su aplicación en las universidades de la Costa Caribe colombiana. Los resultados anteriores evidencian diferencias en las opiniones de los líderes de investigación, lo que permite inferir que las universidades de la Costa Caribe colombiana no tienen un enfoque definido para el desarrollo de la investigación y lo fundamentan tomando en cuenta el predominio de los enfoques estratégico corporativo y en menor medida el social evolutivo.

\section{Referencias}

Bueno, E. (2003). Gestión del conocimiento en universidades y organismos públicos de investigación. Madrid: Universidad Autónoma de Madrid.

Bueno, E. y CIC. (2008). Modelo Intellectus: medición y gestión del capital intelectual, Documentos Intellectus. Madrid. IADE. Universidad Autónoma de Madrid.

Cobo, A. (2006). Modelo de capital intelectual de la Consejería de Educación de la Junta de Andalucía. Indicadores de capital humano y gestión del conocimiento. Tesis Doctoral (Departamento de Economía y Administración de Empresas) Universidad De Málaga.

Da Silva, H., Rodríguez, P., Fernández, C. Y Fernández, J. (2009). La influencia del capital intelectual en la capacidad de innovación de las empresas del Sector de Automoción de la Eurorregión Galicia Norte De Portugal. Vigo.

Seguí, M. (2007). La gestión del capital intelectual en las entidades financieras. Caracterización del capital humano en las cooperativas de crédito. Tesis doctoral. Departament d'Economia i CCSS - UPV. Valencia, España.

Tapscott, D., Ticoll, D. y Lowy, A. (2000). Capital digital. El poder de las redes de negocios. (Trad. M. Condor). Madrid: Taurus digital.

Tapscott, Don (2000) La creación de valor en la economía digital. Buenos Aires: Ediciones Granisa S.A.

Tapscott, D., Lowy, A. \& Ticoll, D. (2000). Digital Capital: Harnessing the Power of Business Webs . Boston: Harvard Business School Press.

Viedma Marti, José María. (2002). Nuevas aportaciones en la construcción del paradigma del capital intelectual. Tomado el 24 de febrero del 2012 de : http://gestiondelcapitalintelectual.com 